\title{
Representations of Multiplier Algebras in Spaces of Completely Bounded Maps
}

\author{
Nico Spronk
}

\begin{abstract}
If $G$ is a locally compact group, then the measure algebra $\mathrm{M}(G)$ and the completely bounded multipliers of the Fourier algebra $\mathrm{M}_{c b} \mathrm{~A}(G)$ can be seen to be dual objects to one another in a sense which generalises Pontryagin duality for abelian groups. We explore this duality in terms of representations of these algebras in spaces of completely bounded maps.
\end{abstract}

This article is intended to give a tour of the growing body of work on representing multiplier algebras in spaces of maps on the $\mathrm{C}^{*}$-algebra $\mathcal{B}(\mathcal{H})$, where $\mathcal{H}$ is a Hilbert space. The ideas here begin in the 1980s with the work of Størmer [37], on representations of measure algebras of abelian groups; work of Ghahramani [12], on a representation of group algebras for general groups; and unpublished work of Haagerup [13], on a representation of completely bounded multipliers of the Fourier algebra of a general locally compact group. Størmer's and Ghahramani's results have been expanded upon and improved in the work of Neufang [22], Neufang, Ruan and Spronk [23] and Smith and Spronk [33]. The results of Haagerup were rediscovered by Spronk $[\mathbf{3 4}, \mathbf{3 5}]$. These ideas are currently being reinterpreted by Neufang, Ruan and Spronk, and may yield results on multipliers of "quantum groups".

Date: March 2, 2005.

2000 Mathematics Subject Classification. Primary 46L07, 22D20, 43A20; Secondary 22D10,22D25. Key words and phrases. Group algebra, Fourier algebra, completely bounded map, extended Haagerup tensor product.

The author was supported by an NSERC PDF. 


\section{Harmonic Analysis}

For any Banach space $\mathcal{X}$ we let $\mathcal{B}(\mathcal{X})$ denote the Banach algebra of bounded linear operators on $\mathcal{X}$.

Let $G$ be a locally compact group. Let, for $1 \leq p \leq \infty, \mathrm{L}^{p}(G)$ denote the space of (almost everywhere equivalence classes) of functions on $G$ which are $p$ integrable with respect to integration over the left Haar measure $\int_{G} \cdots d s$. We will let $\operatorname{M}(G)$ denote the usual measure algebra over $G$, which is the dual of the space of continuous functions vanishing at infinity $\mathcal{C}_{0}(G)$. We note that $\mathrm{M}(G)$ also injects into the dual of the space of bounded continuous functions $\mathcal{C}_{b}(G)$. All of these spaces are discussed in the standard references $[\mathbf{1 6}, \mathbf{2 4}, \mathbf{3 0}]$.

Let $\mathcal{H}$ be a Hilbert space and $\pi: G \rightarrow \mathcal{U}(\mathcal{H})$ be a strongly continuous unitary representation. Then there exists a homomorphism $\pi_{1}: \mathrm{M}(G) \rightarrow \mathcal{B}(\mathcal{H})$ given for each $\mu$ in $\mathrm{M}(G)$ by the vector integral $\int_{G} \pi(s) d \mu(s)$, where the integral may be considered to converge in the weak* topology in $\mathcal{B}(\mathcal{H})$. We identify the group algebra $\mathrm{L}^{1}(G)$ with the ideal of measures in $\mathrm{M}(G)$ which are absolutely continuous with respect to Haar measure. We identify the discrete group algebra $\ell^{1}(G)$ with the closed span of the Dirac measures on $G$. We then let

$$
\mathrm{C}_{\pi}^{*}=\overline{\pi_{1}\left(\mathrm{~L}^{1}(G)\right)}, \quad \mathrm{D}_{\pi}^{*}=\overline{\pi_{1}\left(\ell^{1}(G)\right)} \quad \text { and } \quad \mathrm{M}_{\pi}^{*}=\overline{\pi_{1}(\mathrm{M}(G))}
$$

where closures are each taken in the norm topology of $\mathcal{B}(\mathcal{H})$. We note that $\mathrm{M}_{\pi}^{*}$ is contained in $\mathrm{MC}_{\pi}^{*}$, the multiplier $\mathrm{C}^{*}$-algebra of $\mathrm{C}_{\pi}^{*}$. However, $\mathrm{M}_{\pi}^{*} \neq \mathrm{MC}_{\pi}^{*}$, in general; an example may be found in taking $G=\mathbb{R}$ and $\pi$ to be the left regular representation.

The spaces $\mathrm{A}(G)$ and $\mathrm{B}(G)$ are the Fourier and Fourier-Stieltjes algebras, as introduced in Eymard [11]. If $G$ is abelian with Pontryagin dual group $\widehat{G}$, then the Fourier transform identifies $\mathrm{A}(G) \cong \mathrm{L}^{1}(\widehat{G})$ and $\mathrm{B}(G) \cong \mathrm{M}(\widehat{G})$. Thus $\mathrm{A}(G)$ and $\mathrm{B}(G)$ play roles analogous to $\mathrm{L}^{1}(G)$ and $\mathrm{M}(G)$. The multipliers of $\mathrm{A}(G)$ are given by

$$
\operatorname{MA}(G)=\{u: G \rightarrow \mathbb{C} \mid u v \in \mathrm{A}(G) \text { for each } v \in \mathrm{A}(G)\}
$$

An application of the Closed Graph Theorem shows that each multiplier automatically gives rise to a bounded linear operator $m_{u}: \mathrm{A}(G) \rightarrow \mathrm{A}(G), m_{u} v=u v$. 
Thus $\mathrm{MA}(G)$ is a commutative Banach subalgebra of $\mathcal{B}(\mathrm{A}(G))$. The Fourier algebra $\mathrm{A}(G)$ is the predual of the group von Neumann algebra $\operatorname{VN}(G)$, and as such obtains a natural structure as an operator space, as has been observed by Blecher $[\mathbf{2}]$ and by Effros and Ruan [8]. It thus makes sense to define the completely bounded multipliers

$$
\mathrm{M}_{c b} \mathrm{~A}(G)=\left\{u \in \operatorname{MA}(G) \mid m_{u} \in \mathcal{C B}(\mathrm{A}(G))\right\}
$$

where $\mathcal{C B}(\mathrm{A}(G))$ is the algebra of completely bounded linear maps on $\mathrm{A}(G)$. (For a full exposition of operator spaces and completely bounded maps, we refer the reader to $[\mathbf{9}]$.) The completely bounded multipliers first studied, in a dual context as maps on $\operatorname{VN}(G)$, by de Cannière and Haagerup $[\mathbf{7}]$ and further studied in $[\mathbf{6}, \mathbf{5}]$.

Wendel's Theorem [41] tells us that $\mathrm{M}(G)$ is the multiplier algebra of $\mathrm{L}^{1}(G)$. Thus if $G$ is abelian, we see that $\operatorname{MA}(G)=\mathrm{B}(G)$. In fact, we always have that $\mathrm{B}(G) \subset \mathrm{M}_{c b} \mathrm{~A}(G) \subset \mathrm{MA}(G)$. Losert $[\mathbf{2 1}]$ has shown that $\mathrm{B}(G)=\mathrm{MA}(G)$ exactly when $G$ is amenable (see $[\mathbf{2 5}, \mathbf{3 0}]$ for more on amenable groups). It is also known, due to an unpublished result of Losert, that $\mathrm{B}(G)=\mathrm{M}_{c b} \mathrm{~A}(G)$ exactly when $G$ is amenable; this was also proved by Bożejko [4] in the case that $G$ is discrete. It is unknown if the equality $\mathrm{M}_{c b} \mathrm{~A}(G)=\mathrm{MA}(G)$ characterises the amenability of $G$.

Hence we see that each of $\mathrm{B}(G), \mathrm{M}_{c b} \mathrm{~A}(G)$ and $\mathrm{MA}(G)$ is a reasonable candidate for the "dual object" of $\mathrm{M}(G)$ in the sense that $\mathrm{A}(G)$ is the dual object for $\mathrm{L}^{1}(G)$. In this article we aim to establish a context in which $\mathrm{M}_{c b} \mathrm{~A}(G)$ is the most reasonable dual object. Another context, one which uses similar techniques to ours, is worked out by Walter $[\mathbf{3 9}, \mathbf{4 0}]$. In that work, $\mathrm{B}(G)$ is the appropriate dual object. The relationship between Walter's results and ours seems worthy of deeper study.

Let us finish with a brief note about operator space structures. Though any normed linear space may be endowed with a multitude of operator space structures, there are certain canonical choices, developed in $[\mathbf{9}, \mathbf{2}]$, which are most natural for certain types of normed linear spaces. We have that $\mathrm{B}(G)$ is the dual of the enveloping $\mathrm{C}^{*}$-algebra $\mathrm{C}^{*}(G)$, and hence we will always assign it the standard dual operator space structure. As mentioned above, $\mathrm{A}(G)$ obtains the standard predual operator space structure as the predual of $\mathrm{VN}(G)$. However, it may be shown that this is the same as the operator subspace structure it inherits being a subspace of $\mathrm{B}(G)$. If $\mathcal{V}$ is any operator space, the algebra of completely bounded 
linear maps on it, $\mathcal{C B}(\mathcal{V})$, will be given the standard operator space structure. Any subspace of $\mathcal{C B}(\mathcal{V})$, such as $\mathrm{M}_{c b} \mathrm{~A}(G)$ which imbeds into $\mathcal{C B}(\mathrm{A}(G))$, will inherit the operator subspace structure. Any $\mathrm{C}^{*}$-algebra will have its $\mathrm{C}^{*}$-algebra operator space structure. In particular, any commutative $\mathrm{C}^{*}$-algebra will be a minimal operator space. Any space $\mathrm{L}^{1}(X, \mu)$, for a measure space $(X, \mu)$, will have the maximal operator space structure. If $X$ is further a locally compact topological space we assign its measure space $\mathrm{M}(X)$ the maximal operator space structure. The maximal operator space structure is the standard dual (resp. predual) structure in terms of the dual pairing $\mathcal{C}_{0}(X)^{*} \cong \mathrm{M}(X)$ (resp. $\left.\mathrm{L}^{\infty}(X, \mu)_{*} \cong \mathrm{L}^{1}(X, \mu)\right)$.

\section{Spaces of Completely Bounded Normal Maps}

Let $\mathcal{H}$ be a Hilbert space and let $\mathcal{C B}^{\sigma}(\mathcal{B}(\mathcal{H}))$ denote the space of all normal completely bounded maps on $\mathcal{B}(\mathcal{H})$. The following can be obtained from the representation theorem for completely bounded maps (see $[\mathbf{1 3}, \mathbf{2 6}, \mathbf{9}]$ ) applied to the algebra $\mathcal{K}(\mathcal{H})$, of compact operators on $\mathcal{H}$.

TheOREM 2.1. $[\mathbf{1 3}, \mathbf{3 2}] T \in \mathcal{C B}^{\sigma}(\mathcal{B}(\mathcal{H}))$ if and only if there exist families $\left\{a_{i}\right\}_{i \in I}$ and $\left\{b_{i}\right\}_{i \in I}$ from $\mathcal{B}(\mathcal{H})$, where the cardinality of the index set $I$ and the Hilbertian dimension of $\mathcal{H}$ are related by $|I|=(\operatorname{dim} \mathcal{H})^{2}$, such that for any $x$ in $\mathcal{B}(\mathcal{H})$

$$
T x=\sum_{i \in I} a_{i} x b_{i} \quad \text { and } \quad\|T\|_{c b}=\left\|\sum_{i \in I} a_{i} a_{i}^{*}\right\|^{1 / 2}\left\|\sum_{i \in I} b_{i}^{*} b_{i}\right\|^{1 / 2}
$$

where all series converge in the weak* topology of $\mathcal{B}(\mathcal{H})$.

We thus define the extended Haagerup tensor product $\mathcal{B}(\mathcal{H}) \otimes^{\text {eh }} \mathcal{B}(\mathcal{H})$ by identifying it with $\mathcal{C B}^{\sigma}\left(\mathcal{B}(\mathcal{H})\right.$ ). Hence elements of $\mathcal{B}(\mathcal{H}) \otimes^{\text {eh }} \mathcal{B}(\mathcal{H})$ are (formal) series of elementary tensors $T=\sum_{i \in I} a_{i} \otimes b_{i}$ where $\|T\|_{e h}^{2}=\left\|\sum_{i \in I} a_{i} a_{i}^{*}\right\|\left\|\sum_{i \in I} b_{i}^{*} b_{i}\right\|<\infty$. We identify series $\sum_{i \in I} a_{i} \otimes b_{i}$ and $\sum_{i \in I} a_{i}^{\prime} \otimes b_{i}^{\prime}$ provided $\sum_{i \in I} a_{i} x b_{i}=\sum_{i \in I} a_{i}^{\prime} x b_{i}^{\prime}$ for each $x$ in $\mathcal{B}(\mathcal{H})$. $\mathcal{B}(\mathcal{H}) \otimes^{\text {eh }} \mathcal{B}(\mathcal{H})$ is a Banach algebra when the multiplication is induced by its identification with $\mathcal{C B}^{\sigma}(\mathcal{B}(\mathcal{H}))$, so on elementary tensors we have multiplication $(a \otimes b) \circ(c \otimes d)=a c \otimes d b$. In this context, we note that the Haagerup tensor product $\mathcal{B}(\mathcal{H}) \otimes^{h} \mathcal{B}(\mathcal{H})$ is the $\|\cdot\|_{c b}$-closure of the space of elementary operators on $\mathcal{B}(\mathcal{H})$. Moreover, each $T$ in $\mathcal{B}(\mathcal{H}) \otimes^{h} \mathcal{B}(\mathcal{H})$ can be represented $T=\sum_{i=1}^{\infty} a_{i} \otimes b_{i}$ where the series $\sum_{i=1}^{\infty} a_{i} a_{i}^{*}$ and $\sum_{i=1}^{\infty} b_{i}^{*} b_{i}$ converge uniformly. See $[\mathbf{3 2}]$ for this description of $\mathcal{B}(\mathcal{H}) \otimes^{h} \mathcal{B}(\mathcal{H})$. 
If $F \in \mathcal{B}(\mathcal{H})^{*}$ we define the left and right slice maps $L_{F}, R_{F}: \mathcal{B}(\mathcal{H}) \otimes^{\text {eh }} \mathcal{B}(\mathcal{H}) \rightarrow$ $\mathcal{B}(\mathcal{H})$ by

$$
L_{F} T=\sum_{i \in I} F\left(a_{i}\right) b_{i} \quad \text { and } \quad R_{F} T=\sum_{i \in I} F\left(b_{i}\right) a_{i}
$$

where $T=\sum_{i \in I} a_{i} \otimes b_{i}$. That $L_{F}$ and $R_{F}$ are well defined operators and that these series converge in norm is shown in $[35]$. The following is from $[\mathbf{3 4}, \mathbf{3 5}]$, which is adapted from $[\mathbf{3}, \mathbf{3 2}]$.

TheOREM 2.2. If $\mathcal{V}$ and $\mathcal{W}$ are closed subspaces of $\mathcal{B}(\mathcal{H})$ and $T \in \mathcal{B}(\mathcal{H}) \otimes^{\text {eh }}$ $\mathcal{B}(\mathcal{H})$ then the following are equivalent:

(i) $L_{F} T \in \mathcal{W}$ and $R_{F} T \in \mathcal{V}$ for each $F$ in $\mathcal{B}(\mathcal{H})^{*}$.

(ii) $L_{\omega} T \in \mathcal{W}$ and $R_{\omega} T \in \mathcal{V}$ for each $\omega$ in $\mathcal{B}(\mathcal{H})_{*}$.

(iii) there exist families $\left\{v_{i}\right\}_{i \in I}$ from $\mathcal{V}$ and $\left\{w_{i}\right\}_{i \in I}$ from $\mathcal{W}$ such that $T=$ $\sum_{i \in I} v_{i} \otimes w_{i}$ and $\|T\|_{e h}=\left\|\sum_{i \in I} v_{i} v_{i}^{*}\right\|^{1 / 2}\left\|\sum_{i \in I} w_{i}^{*} w_{i}\right\|^{1 / 2}$.

The space of all $T$ satisfying any of the above conditions is thus denoted $\mathcal{V} \otimes^{e h} \mathcal{W}$. The spaces $\mathcal{V} \otimes^{e h} \mathcal{W}$, as well as extended Haagerup tensor products of multiple operator spaces, are also developed by Effros and Ruan [10], using different methods. Their goal in that article is to study "operator convolution algebras", a goal similar to ours in the present article.

Let us summarise some properties of the extended Haagerup tensor product.

THEOREM 2.3. (i) $[\mathbf{3}, \mathbf{1 0}]$ If $\mathcal{V}$ and $\mathcal{W}$ are dual operator spaces with respective preduals $\mathcal{V}_{*}$ and $\mathcal{W}_{*}$, then $\mathcal{V} \otimes^{e h} \mathcal{W} \cong\left(\mathcal{V}_{*} \otimes^{h} \mathcal{W}_{*}\right)^{*}$

(ii) $[33,1]$ If $\mathcal{A}$ and $\mathcal{B}$ are each subalgebras of $\mathcal{B}(\mathcal{H})$ then $\mathcal{A} \otimes^{\text {eh }} \mathcal{B}$ is a subalgebra of $\mathcal{B}(\mathcal{H}) \otimes^{\text {eh }} \mathcal{B}(\mathcal{H})$. If $\mathcal{I}$ is a left ideal of $\mathcal{A}$ and $\mathcal{J}$ is a right ideal of $\mathcal{B}$, then $\mathcal{I} \otimes^{\text {eh }} \mathcal{J}$ is a left ideal of $\mathcal{A} \otimes^{e h} \mathcal{B}$.

(iii) $[32,3,13]$ If $\mathcal{M}$ is a locally cyclic $C^{*}$-subalgebra of $\mathcal{B}(\mathcal{H})$, i.e. for every finite dimensional subspace $\mathcal{L}$ of $\mathcal{H}$ there is $\xi \in \mathcal{H}$ such that $\overline{\mathcal{M} \xi} \supset \mathcal{L}$, then each $\mathcal{M}$-bimodule map on $\mathcal{B}(\mathcal{H})$ is automatically completely bounded. Moreover, there is a natural identification between the space of normal $\mathcal{M}$-bimodule maps $\mathcal{B}_{\mathcal{M}}^{\sigma}(\mathcal{B}(\mathcal{H}))$ and $\mathcal{M}^{\prime} \otimes^{\text {eh }} \mathcal{M}^{\prime}$, where $\mathcal{M}^{\prime}$ is the commutant of $\mathcal{M}$ in $\mathcal{B}(\mathcal{H})$.

Let us expose these ideas by introducing a class of commutative Banach algebras which has been studied in $[\mathbf{3 4}, \mathbf{3 5}, \mathbf{3 1}]$. If $X$ is a locally compact Hausdorff space, 
then, by Grothendieck's inequality, the algebra $\mathrm{V}_{0}(X)=\mathcal{C}_{0}(X) \otimes^{h} \mathcal{C}_{0}(X)$ is exactly the algebra $\mathcal{C}_{0}(X) \otimes^{\gamma} \mathcal{C}_{0}(X)$ (projective tensor product) studied by Varopoulos $[\mathbf{3 8}]$. We thus dub it the Varopoulos algebra. The extended Varopoulos algebras are given by

$$
\mathrm{V}^{0}(X)=\mathcal{C}_{0}(X) \otimes^{e h} \mathcal{C}_{0}(X) \quad \text { and } \quad \mathrm{V}^{b}(X)=\mathcal{C}_{b}(X) \otimes^{e h} \mathcal{C}_{b}(X)
$$

An element $w$ of $\mathrm{V}^{b}(X)$ can be written $w=\sum_{i \in I} \varphi_{i} \otimes \psi_{i}$, where we have that $\left\|\sum_{i \in I}\left|\varphi_{i}\right|^{2}\right\|_{\infty}\left\|\sum_{i \in I}\left|\psi_{i}\right|^{2}\right\|_{\infty}<\infty$. These series can be taken to converge pointwise in $\ell^{\infty}(X)$. Thus we may consider $w$ to be a function on $X \times X$ by letting $w(x, y)=\sum_{i \in I} \varphi_{i}(x) \psi_{i}(y)$ for each $(x, y)$. As pointed out in [31], $w$ need not be continuous on $X \times X$. However, for any fixed $x$ in $X$, pointwise slices $w(\cdot, x)$ and $w(x, \cdot)$ are in $\mathcal{C}_{b}(X)$ (and in $\mathcal{C}_{0}(X)$ if $w \in \mathrm{V}^{0}(X)$ ). If $(X, \mu)$ is any measure space, we let $\mathrm{V}^{\infty}(X, \mu)=\mathrm{L}^{\infty}(X, \mu) \otimes^{e h} \mathrm{~L}^{\infty}(X, \mu)$. Similarly as above, we may consider elements of $\mathrm{V}^{\infty}(X, \mu)$ to be functions on $X \times X$, but defined only up to locally marginally null sets. We note that if $(X, \mu)$ is further assumed to satisfy the Radon-Nikodym Theorem, so $\mathrm{L}^{1}(X, \mu)^{*} \cong \mathrm{L}^{\infty}(X, \mu)$, then $\mathrm{V}^{\infty}(X, \mu)$ is naturally isomorphic to each of

$$
\left(\mathrm{L}^{1}(X, \mu) \otimes^{h} \mathrm{~L}^{1}(X, \mu)\right)^{*} \quad \text { and } \quad \mathcal{B}_{\mathrm{L}^{\infty}(X, \mu)}^{\sigma}\left(\mathcal{B}\left(\mathrm{L}^{2}(X, \mu)\right)\right)
$$

where $\mathrm{L}^{\infty}(X, \mu)$ is identified with the maximal abelian subalgebra of multiplication operators on $\mathrm{L}^{2}(X, \mu)$. In particular, if $\mu$ is counting measure on $X$, then $\mathrm{V}^{\infty}(X, \mu)=\ell^{\infty}(X) \otimes^{e h} \ell^{\infty}(X)$ is shown in $[\mathbf{3 4}, 35]$ to be the algebra of Schur multipliers on $\mathcal{B}\left(\ell^{2}(X)\right)$. Variants of this fact are established by Hladnik [17] and Pisier [27]. This justifies referring to the space $\mathrm{V}^{\infty}(X, \mu)$, for a measure space $(X, \mu)$, as the space of measurable Schur multipliers.

\section{Representations of $\mathrm{M}(G)$}

Let $G$ be a locally compact group, $\mathcal{H}$ be a Hilbert space and $\pi: G \rightarrow \mathcal{U}(\mathcal{H})$ be a strongly continuous representation. The representation $\pi_{1}: \mathrm{M}(G) \rightarrow \mathcal{B}(\mathcal{H})$ may retain some information about the Banach algebra $\mathrm{M}(G)$ or its ideal $\mathrm{L}^{1}(G)$. However, it is shown by Ghahramani [12] that it is impossible for there to exist any isometric homomorphism from $\mathrm{L}^{1}(G)$ into $\mathcal{B}(\mathcal{H})$. Thus it is intriguing to note that $\pi$ still can be exploited to give a representation of $\mathrm{M}(G)$ which retains some spatial information. 
Let $\gamma_{\pi}: G \rightarrow \mathcal{B}(\mathcal{H}) \otimes^{e h} \mathcal{B}(\mathcal{H})$ be given by

$$
\gamma_{\pi}(s)=\pi(s) \otimes \pi(s)^{*} .
$$

By the multiplication on $\mathcal{B}(\mathcal{H}) \otimes^{e h} \mathcal{B}(\mathcal{H}) \cong \mathcal{C B}^{\sigma}(\mathcal{B}(\mathcal{H}))$, $\gamma_{\pi}$ is a homomorphism. We can view the continuity of $\gamma_{\pi}$ in two ways:

(i) $\mathcal{B}(\mathcal{H}) \otimes^{e h} \mathcal{B}(\mathcal{H})$ has a weak* topology given by the predual $\mathcal{B}(\mathcal{H})_{*} \otimes^{h} \mathcal{B}(\mathcal{H})_{*}$. $\gamma_{\pi}$ is continuous in this weak ${ }^{*}$ topology.

(ii) For each $s$ in $G, \gamma_{\pi}(s)(\mathcal{K}(\mathcal{H})) \subset \mathcal{K}(\mathcal{H})$. The map $\left.s \mapsto \gamma_{\pi}(s)\right|_{\mathcal{K}(\mathcal{H})}$ from $G$ to $\mathcal{C B}(\mathcal{K}(\mathcal{H}))$ is continuous when $\mathcal{C B}(\mathcal{K}(\mathcal{H}))$ has the point-norm topology.

We can use $\gamma_{\pi}$ to define a map $\Gamma_{\pi}: \mathrm{M}(G) \rightarrow \mathcal{B}(\mathcal{H}) \otimes^{\text {eh }} \mathcal{B}(\mathcal{H})$ by letting for each $\mu$ in $\mathrm{M}(G)$,

$$
\Gamma_{\pi}(\mu)=\int_{G} \gamma_{\pi}(s) d \mu(s)=\int_{G} \pi(s) \otimes \pi(s)^{*} d \mu(s) .
$$

This vector-valued integral can be interpreted in one of two ways. Using (i), we can view (3.1) as converging in the weak* topology of $\mathcal{B}(\mathcal{H}) \otimes^{\text {eh }} \mathcal{B}(\mathcal{H})$, i.e. for each $x=\sum_{i=1}^{\infty} \omega_{i} \otimes v_{i}$ in $\mathcal{B}(\mathcal{H})_{*} \otimes^{h} \mathcal{B}(\mathcal{H})_{*}$ we have

$$
\left\langle\Gamma_{\pi} \mu, x\right\rangle=\int_{G}\left\langle\pi(s) \otimes \pi(s)^{*}, x\right\rangle d \mu(s)=\sum_{i=1}^{\infty} \int_{G}\left\langle\pi(s), \omega_{i}\right\rangle\left\langle\pi(s)^{*}, v_{i}\right\rangle d \mu(s) .
$$

On the other hand, by (ii), we can obtain Bochner integrals pointwise on $\mathcal{K}(\mathcal{H})$, i.e. for each $k$ in $\mathcal{K}(\mathcal{H})$ we let

$$
\tilde{\gamma}_{\pi}(\mu) k=\int_{G} \pi(s) k \pi(s)^{*} d \mu(s) .
$$

Then $\tilde{\gamma}_{\pi}: \mathrm{M}(G) \rightarrow \mathcal{B}(\mathcal{K}(\mathcal{H}))$ is a bounded homomorphism whose range may be verified to be in $\mathcal{C B}(\mathcal{K}(\mathcal{H}))$. We can then view each operator $\Gamma_{\pi}(\mu): \mathcal{B}(\mathcal{H}) \rightarrow \mathcal{B}(\mathcal{H})$ as the second Banach space adjoint $\tilde{\gamma}_{\pi}(\mu)^{* *}$. Thus $\Gamma_{\pi}: \mathrm{M}(G) \rightarrow \mathcal{B}(\mathcal{H}) \otimes^{\text {eh }} \mathcal{B}(\mathcal{H})$ is a homomorphism. We note that this fact is established in [33], using (i) above; the proof is complicated by the fact that the Banach algebra $\mathcal{B}(\mathcal{H}) \otimes^{\text {eh }} \mathcal{B}(\mathcal{H})$ does not have weak* continuous multiplication on the right, in general.

We now consider the left regular representation $\lambda$. The next result is due to Størmer [37] in the case that $G$ is abelian, and due to Gharamani [12] in general. It is recognised by Neufang $[\mathbf{2 2}]$ that the image is one of completely bounded maps.

TheOREM 3.1. The map $\Gamma_{\lambda}: \mathrm{M}(G) \rightarrow \mathcal{C B}^{\sigma}\left(\mathcal{B}\left(\mathrm{L}^{2}(G)\right)\right)$ is a completely isometric representation. 
One of the crucial calculations that makes Theorem 3.1 work is that if we let $\left\{M_{\varphi}: \varphi \in \mathrm{L}^{\infty}(G)\right\}$ be the algebra of multiplication operators on $\mathrm{L}^{2}(G)$, then for each $\mu$ in $\mathrm{M}(G)$ and $\varphi$ in $\mathrm{L}^{\infty}(G)$ we have that $\Gamma_{\lambda}(\mu) M_{\varphi}=M_{\mu * \varphi}$, so $\Gamma_{\lambda}$ extends the action of $\mathrm{M}(G)$ on $\mathrm{L}^{\infty}(G)$.

We note that $\Gamma_{\lambda}(\mathrm{M}(G)) \subset \mathrm{VN}(G) \otimes^{\text {eh }} \mathrm{VN}(G)$. In fact, it is shown in [23] that $\Gamma_{\lambda}: \mathrm{M}(G) \rightarrow \mathrm{VN}(G) \otimes^{e h} \mathrm{VN}(G)$ is the adjoint of the map $\left(\Gamma_{\lambda}\right)_{*}: \mathrm{A}(G) \otimes^{h} \mathrm{~A}(G) \rightarrow$ $\mathcal{C}_{0}(G)$ given by $\left(\Gamma_{\lambda}\right)_{*}(u \otimes v)=u \check{v}$, where $\check{v}(s)=v\left(s^{-1}\right)$. It follows from Theorem 3.1 that $\left(\Gamma_{\lambda}\right)_{*}$ is a complete quotient map.

We also note that Neufang has extended Theorem 3.1 to obtain a map from the dual space of left uniformly continuous bounded functions $\mathcal{L U C}(G)^{*}$ (see [20], for information on this algebra) to $\mathcal{C B}\left(\mathcal{B}\left(\mathrm{L}^{2}(G)\right)\right)$, which extends $\Gamma_{\lambda}$. See $[\mathbf{2 2}]$.

Smith and Spronk [33] study the ranges of the maps $\Gamma_{\pi}$, in general.

Proposition 3.2. (i) $\Gamma_{\pi}(\mathrm{M}(G)) \subset \mathrm{M}_{\pi}^{*} \otimes^{e h} \mathrm{M}_{\pi}^{*} \subset \mathrm{MC}_{\pi}^{*} \otimes^{e h} \mathrm{MC}_{\pi}^{*}$

(ii) $\Gamma_{\pi}\left(\mathrm{L}^{1}(G)\right) \subset \mathrm{C}_{\pi}^{*} \otimes^{e h} \mathrm{C}_{\pi}^{*}$

(iii) $\Gamma_{\pi}\left(\ell^{1}(G)\right) \subset \mathrm{D}_{\pi}^{*} \otimes^{h} \mathrm{D}_{\pi}^{*}$

We see then that for a discrete measure $\mu$ in $\ell^{2}(G)$ that $\Gamma_{\pi}(\mu)$ can be approximated uniformly by elementary operators. It is further investigated in [33] when this can be done for arbitrary measures.

THEOREM 3.3. The following are equivalent:

(i) $\Gamma_{\pi}(\mathrm{M}(G)) \subset \mathrm{M}_{\pi}^{*} \otimes^{h} \mathrm{M}_{\pi}^{*}$

(ii) $\Gamma_{\pi}\left(\mathrm{L}^{1}(G)\right) \subset \mathrm{C}_{\pi}^{*} \otimes^{h} \mathrm{C}_{\pi}^{*}$

(iii) $\pi: G \rightarrow \mathcal{U}(\mathcal{H})$ is norm continuous.

We note that in the case of abelian groups, that (iii) implies (i), above, is shown by Størmer [37]. In [33] the following fact was used.

Proposition 3.4. The following are equivalent:
(i) $\pi$ is norm continuous
(ii) $\mathrm{C}_{\pi}^{*}$ is unital
(iii) $\mathrm{C}_{\pi}^{*}=\mathrm{D}_{\pi}^{*}$.

Condition (iii) allows us to think of norm continuity of $\pi$ as being a "discreteness" condition. Note that in the case that $G$ is abelian, with dual group $\widehat{G}$, Proposition 3.4 implies that $\operatorname{supp}(\pi)$ is compact. If $G$ is compact, with dual object $\widehat{G}$, then Proposition 3.4 implies that $\operatorname{supp}(\pi)$ is finite. These results are established 
for the class of direct products of vector groups by compact groups by Kallman [19], using different methods.

\section{Representations of $\mathrm{M}_{c b} \mathrm{~A}(G)$}

In [15], Herz defines $\mathrm{B}_{2}(G)$ to be the space of Schur multipliers $u$ on $\mathcal{B}\left(\ell^{2}(G)\right)$ which are continuous and satisfy the following invariance condition: $u(s, t)=$ $u(s r, t r)$ for all $s, t, r$ in $G$. Bożejko and Fendler [5] prove that $\mathrm{B}_{2}(G)=\mathrm{M}_{c b} \mathrm{~A}(G)$. There are similar results, due to Haagerup [14] and Jolissaint [18]. Thus there is a strong link between $\mathrm{M}_{c b} \mathrm{~A}(G)$ and Schur multipliers.

The main result relating $\mathrm{M}_{c b} \mathrm{~A}(G)$ with measurable Schur multipliers follows. It can be found in the present form in $[\mathbf{3 4}, \mathbf{3 5}]$, but there is an isometric version due to Haagerup [13]. Below, we let $m$ denote the left Haar measure on $G$ and

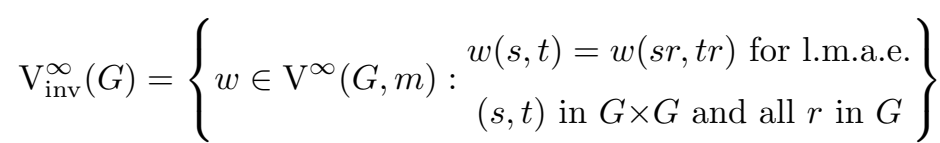

where l.m.a.e. means "locally marginally almost every".

Theorem 4.1. If $u \in \mathrm{M}_{c b} \mathrm{~A}(G)$, then $N u$, given for $(s, t)$ in $G \times G$ by

$$
N u(s, t)=u\left(s t^{-1}\right)
$$

defines an element of $\mathrm{V}^{\infty}(G, m)$. Moreover the map $N: \mathrm{M}_{c b} \mathrm{~A}(G) \rightarrow \mathrm{V}^{\infty}(G, m)$ is a complete isometry whose image is $\mathrm{V}_{\mathrm{inv}}^{\infty}(G)$.

Using slice maps on each $N u$, it can be established that

$$
N\left(\mathrm{M}_{c b} \mathrm{~A}(G)\right) \subset \mathrm{V}^{b}(G)=\mathcal{C}_{b}(G) \otimes^{e h} \mathcal{C}_{b}(G)
$$

which is an analogue of Proposition 3.2 (i). However, we can further establish that

$$
N\left(\mathrm{M}_{c b} \mathrm{~A}(G)\right) \subset{\overline{\mathrm{M}_{c b} \mathrm{~A}(G)}}^{\|\cdot\|_{\infty}} \otimes^{e h}{\overline{\mathrm{M}_{c b} \mathrm{~A}(G)}}^{\|\cdot\|_{\infty}} \subset \mathcal{W} \mathcal{A} \mathcal{P}(G) \otimes^{e h} \mathcal{W} \mathcal{A} \mathcal{P}(G)
$$

where $\mathcal{W} \mathcal{A} \mathcal{P}(G)$ is the algebra of weakly almost periodic functions on $G$. We note that the inclusion $\mathrm{M}_{c b} \mathrm{~A}(G) \subset \mathcal{W} \mathcal{A} \mathcal{P}(G)$ was established by $\mathrm{Xu}[\mathbf{4 2}, 43]$.

We note that as a Corollary of Theorem 4.1, we obtain a characterisation of the predual $\mathrm{Q}(G)$ of $\mathrm{M}_{c b} \mathrm{~A}(G)$, whose existence was established by de Cannière and Haagerup [7]: there is a map from $\mathrm{L}^{1}(G) \otimes^{h} \mathrm{~L}^{1}(G)$ to $\mathrm{Q}(G)$ given on elementary tensors by $f \otimes g \mapsto f * g$, which is a complete quotient. Thus it follows from Losert's 
result characterising when $\mathrm{M}_{c b} \mathrm{~A}(G)=\mathrm{B}(G)$ that $\mathrm{C}^{*}(G)$ is a quotient of $\mathrm{L}^{1}(G) \otimes^{h}$ $\mathrm{L}^{1}(G)$ exactly when $G$ is amenable. An equivalent form of this result appears to go back to Grothendieck, and is exposed by Racher [28].

\section{Parallels}

We now strive to compare the results of the previous two sections. Below we identify $\mathrm{L}^{\infty}(G)$ with the algebra of multiplication operators on $\mathrm{L}^{2}(G)$. If $\mathcal{M}$ is an von Neumann subalgebra of $\mathcal{B}(\mathcal{H})$, we denote the space of completely bounded normal $\mathcal{M}$-bimodule maps on $\mathcal{B}(\mathcal{H})$ by $\mathcal{C B}_{\mathcal{M}}^{\sigma}(\mathcal{B}(\mathcal{H}))$. Note that by Theorem 2.3 (iii) this is always $\mathcal{M}^{\prime} \otimes^{e h} \mathcal{M}^{\prime}$. Moreover, also by Theorem 2.3 (iii), if $\mathcal{M}$ is locally cyclic, then $\mathcal{C B}_{\mathcal{M}}^{\sigma}(\mathcal{B}(\mathcal{H}))=\mathcal{B}_{\mathcal{M}}^{\sigma}(\mathcal{B}(\mathcal{H}))$.

Let $\rho: G \rightarrow \mathcal{U}\left(\mathrm{L}^{2}(G)\right)$ be the right regular representation. Since $\rho$ is similar to the left regular representation $\lambda$, we have that Theorem 3.1 holds for $\Gamma_{\rho}: \mathrm{M}(G) \rightarrow$ $\mathcal{C B}^{\sigma}\left(\mathcal{B}\left(\mathrm{L}^{2}(G)\right)\right)$.

THEOREM 5.1. The ranges of $\Gamma_{\rho}$ and $N$ can be characterised by the following invariance conditions:

(i) $[\mathbf{2 2}] \Gamma_{\rho}(\mathrm{M}(G))=\left\{T \in \mathcal{C B}_{\mathrm{VN}(G)}^{\sigma}\left(\mathcal{B}\left(\mathrm{L}^{2}(G)\right)\right): T \mathrm{~L}^{\infty}(G) \subset \mathrm{L}^{\infty}(G)\right\}$.

(ii) $\left[\right.$ 23] $N\left(\mathrm{M}_{c b} \mathrm{~A}(G)\right)=\left\{T \in \mathcal{C B}_{\mathrm{L}^{\infty}(G)}^{\sigma}\left(\mathcal{B}\left(\mathrm{L}^{2}(G)\right)\right): T \mathrm{VN}(G) \subset \mathrm{VN}(G)\right\}$.

This is very strong evidence that Theorems 3.1 and 4.1 are two examples of the same phenomenon - to wit, there may be a Kac algebra or quantum group theorem from which these results can be deduced. This becomes even more apparent if we consider the case that $G$ is abelian, with dual group $\widehat{G}$. In analogy with maps $\rho, N$ and $\Gamma_{\rho}$ above, we define $\hat{\rho}: \widehat{G} \rightarrow \mathcal{U}\left(\mathrm{L}^{2}(\widehat{G})\right), \widehat{N}: \mathrm{B}(\widehat{G})=\mathrm{M}_{c b} \mathrm{~A}(\widehat{G}) \rightarrow$ $\mathcal{C B}^{\sigma}\left(\mathcal{B}\left(\mathrm{L}^{2}(\widehat{G})\right)\right)$ and $\widehat{\Gamma}_{\hat{\rho}}: \mathrm{M}(\widehat{G}) \rightarrow \mathcal{C B}^{\sigma}\left(\mathcal{B}\left(\mathrm{L}^{2}(\widehat{G})\right)\right)$. We can adjust the Plancherel unitary $U: \mathrm{L}^{2}(G) \rightarrow \mathrm{L}^{2}(\widehat{G})$ in such a way that

$$
\operatorname{Ad} U(\operatorname{VN}(G))=\mathrm{L}^{\infty}(\widehat{G}) \quad \text { and } \quad \operatorname{Ad} U\left(\mathrm{~L}^{\infty}(G)\right)=\operatorname{VN}(\widehat{G})
$$

where $\operatorname{Ad} U x=U x U^{*}$ for each $x$ in $\mathcal{B}\left(\mathrm{L}^{2}(G)\right)$. The following is in [23].

Theorem 5.2. (i) $\operatorname{Ad} U \Gamma_{\rho}(\mathrm{M}(G)) \operatorname{Ad} U^{*}=\widehat{N}(\mathrm{~B}(\widehat{G}))$

(ii) $\operatorname{Ad} U N(\mathrm{~B}(G)) \operatorname{Ad} U^{*}=\widehat{\Gamma}_{\hat{\rho}}(\mathrm{M}(\widehat{G}))$ 
Let us close by illustrating a final parallel. If $G$ is discrete, then it follows from Proposition 3.2 (iii) that

$$
\Gamma_{\lambda}\left(\ell^{1}(G)\right) \subset \mathrm{C}_{r}^{*}(G) \otimes^{h} \mathrm{C}_{r}^{*}(G)
$$

where $\mathrm{C}_{r}^{*}(G)=\mathrm{C}_{\lambda}^{*}$ is the reduced $\mathrm{C}^{*}$-algebra of $G$. An analogue ought to hold then for compact groups in the context of the Fourier algebra. It is shown in $[\mathbf{3 4}, \mathbf{3 5}]$ that the canonical map from $G$ to its almost periodic compactification $G^{\text {ap }}$ induces a complete isometry $\mathrm{A}\left(G^{\mathrm{ap}}\right) \hookrightarrow \mathrm{M}_{c b} \mathrm{~A}(G)$. The result (i), below, is due to Spronk and Turowska [36] and its extension, (ii), is in [34]. If $G$ is a compact abelian group, then (i) is due to Varopoulos [38]. An equivalent result to (ii) has also been discovered by Racher $[\mathbf{2 9}]$.

TheOREM 5.3. (i) If $G$ is compact then $N(\mathrm{~A}(G)) \subset \mathrm{V}_{0}(G)=\mathcal{C}_{b}(G) \otimes^{h} \mathcal{C}_{b}(G)$.

(ii) $N\left(\mathrm{~A}\left(G^{\text {ap }}\right)\right) \subset \mathcal{C}_{b}(G) \otimes^{h} \mathcal{C}_{b}(G)$

We note that in analogy with Theorem 3.3 , it has been shown in $[\mathbf{3 6}, \mathbf{3 4}, \mathbf{3 5}$ ] that the inclusion $N(\mathrm{~A}(G)) \subset \mathrm{V}_{0}(G)=\mathcal{C}_{0}(G) \otimes{ }^{h} \mathcal{C}_{0}(G)$ implies that $G$ is compact.

\section{References}

[1] D. P. Blecher. Geometry of the tensor product of $\mathrm{C}^{*}$-algebras. Math. Proc. Cambridge Phil. Soc., 104(1):119-127, 1988.

[2] D. P. Blecher. The standard dual of an operator space. Pacific Math. J., 153(1):15-30, 1992.

[3] D. P. Blecher and R. R. Smith. The dual of the Haagerup tensor product. J. London Math. Soc., 45(2):126-144, 1992.

[4] M. Bożejko. Positive definite bounded matrices and a characterization of amenable groups. Proc. Amer. Math. Soc., 95(3):357-360, 1985.

[5] M. Bożejko and G. Fendler. Herz-Schur multipliers and completely bounded multipliers of the Fourier algebra of a locally compact group. Boll. Un. Mat. Ital. A (6), 3(2):297-302, 1984.

[6] M. Cowling and U. Haagerup. Completely bounded multipliers of the Fourier algebra of a simple Lie group of real rank 1. Invent. Math., 96:507-549, 1989.

[7] J. de Cannière and U. Haagerup. Multipliers of the Fourier algebras of some simple Lie groups and their discrete subgroups. Amer. J. Math., 107:455-500, 1984.

[8] E. G. Effros and Z.-J. Ruan. A new approach to operator spaces. Canad. Math. Bull., 34:329337, 1991.

[9] E. G. Effros and Z.-J. Ruan. Operator Spaces, volume 23 of London Math. Soc., New Series. Claredon Press, Oxford Univ. Press, New York, 2000. 
[10] E. G. Effros and Z.-J. Ruan. Operator convolution algebras: an approach to quantum groups. To appear in J. Operator Theory., 2002.

[11] P. Eymard. L'algèbre de Fourier d'un groupe localement compact. Bull. Soc. Math. France, 92:181-236, 1964.

[12] F. Ghahramani. Isometric representations of $\mathrm{M}(\mathrm{G})$ on $\mathcal{B}(\mathcal{H})$. Glasgow Math. J., 23:119-122, 1982.

[13] U. Haagerup. Decomposition of completely bounded maps on operator algebras. Unpublished, 1980.

[14] U. Haagerup. Group $\mathrm{C}^{*}$-algebras without the completely bounded approximation property. Unpublished, 1988.

[15] C. S. Herz. Une généralisation de la notion transformée de Fourier-Stieltjes. Ann. Inst. Fourier (Grenoble), 24(3):145-157, 1974.

[16] E. Hewitt and K. A. Ross. Abstract Harmonic Analysis I, volume 115 of Die Grundlehern der mathemarischen Wissenschaften. Springer, New York, second edition, 1979.

[17] M. Hladnik. Compact Schur multipliers. Proc. Amer. Math. Soc., 128:2585-2591, 2000.

[18] P. Jolissaint. A characterization of completely bounded multipliers of the Fourier algebras. Colloq. Math., 63:311-313, 1992.

[19] R. R. Kallman. A characterization of uniformly continuous unitary representations of connected locally compact groups. Michigan Math. J., 16:257-263, 1969.

[20] A. T.-M. Lau. Continuity of Arens multiplication on the dual space of bounded uniformly continuous functions on locally compact groups and topological semigroups. Math. Proc. Camb. Phil. Soc., 99:273-283, 1986.

[21] V. Losert. Properties of the Fourier algebra that are equivalent to amenability. Proc. Amer. Math. Soc., 92(3):347-353, 1984.

[22] M. Neufang. Isometric representations of convolution algebras as completely bounded module homomorphisms and a characterization of the measure algebra. Unpublished, 2001.

[23] M. Neufang, Z.-J. Ruan, and N. Spronk. Completely isometric representations of measure algebras and of completely bounded multipliers of Fourier algebras. In preparation, 2004.

[24] T. W. Palmer. Banach Algebras and the General Theory of *-algebras, Vol. I Algebras and Banach Algebras, volume 49 of Encyclopedia of Mathematics and it Applications. Cambridge University Press, Cambridge, New York, 1994.

[25] A. T. Paterson. Amenability, volume 29 of Mathematical Surveys and Monographs. American Mathematical Society, Providence, RI, 1988.

[26] V. I. Paulsen. Every completely polynomially bounded operator is similar to a contraction. J. Funct. Anal., 55:1-17, 1984.

[27] G. Pisier. Multipliers and lacunary sets in non-amenable groups. Amer. J. Math., 117:337376, 1995.

[28] G. Racher. A proposition of Grothendieck revisited. In Banach Space Theory and Its Applications, volume 991 of Lec. Notes in Math., pages 215-227, Berlin Heidelberg, 1983. Springer. 
[29] G. Racher. The nuclear multipiers from $L^{1}(G)$ into $L^{\infty}(G)$. J. Funct. Anal., 122:279-306, 1994.

[30] V. Runde. Lectures on Amenability, volume 1774 of Lec. Notes in Math. Springer, Berlin Heidelberg, 2002.

[31] V. Shulman and L. Turowska. Operator synthesis. 1. synthetic sets, bilattices and tensor algebras. Preprint. To appear in J. Funct. Anal. Also see ArXiv math.FA/030820, 2003.

[32] R. R. Smith. Completely bounded module maps and the Haagerup tensor product. J. Funct. Anal., 102(1):156-175, 1991.

[33] R. R. Smith and N. Spronk. Representations of group algebras in spaces of completely bounded maps. Preprint. To appear in Indiana Univ. Math. J. Also see ArXiv math.FA/0310421, 2003.

[34] N. Spronk. On Multipliers of the Fourier Algebra of a Locally Compact Group. PhD thesis, University of Waterloo, Waterloo, Ontario, Canada, 2002.

[35] N. Spronk. Measurable Schur multipliers and completely bounded multipliers of the Fourier algebra. To appear in Proc. London Math. Soc. Also see ArXiv math.FA/0210304, 2003.

[36] N. Spronk and L. Turowska. Spectral synthesis and operator synthesis for compact groups. J. London Math. Soc., 66:361-376, 2002.

[37] E. Størmer. Regular Abelian Banach algebras of linear maps of operator algebras. J. Funct. Anal., 37:331-373, 1980.

[38] N. Th. Varopoulos. Tensor algebras and harmonic analysis. Acta. Math., 119:51-112, 1967.

[39] M. E. Walter. Dual algebras. Math. Scand., 58:77-104, 1986.

[40] M. E. Walter. On a new method for defining the norm of Fourier-Stietjes algebras. Pacific J. Math., 137:209-223, 1989.

[41] J. G. Wendell. Left centralizers and isomorphisms of group algebras. Pacific J. Math., 2:251$261,1952$.

[42] G. Xu. Multipiers of the Algebras $A_{p}(G)$. PhD thesis, SUNY Buffalo, NY, 1995.

[43] G. Xu. Herz-Schur multipliers and weakly almost periodic functions on locally compact groups. Trans. Amer. Math. Soc., 349:2525-2536, 1997. 